Instituto Internacional de Investigación y Desarrollo Tecnológico Educativo INDTEC, C.A.

DOI: https://doi.org/10.29394/scientific.issn.2542-2987.2017.2.5.3.49-65

OAI-PMH: http://www.indteca.com/ojs/index.php/Revista Scientific/oai

\title{
Ecología Profunda y Transdisciplinaria en la Conservación de Ambientes Educativos
}

Autora: Janry Mercedes Nádales Pérez

Universidad Pedagógica Experimental Libertador, UPEL

janrynadales@gmail.com

Barinas, Venezuela

\section{Resumen}

El presente trabajo de investigación tuvo como propósito general Desarrollar acciones de ecología profunda y Transdisciplinaria en la preservación de ambientes educativos del Liceo Nacional Bolivariano Dr. Carlos María González Bona, ubicado en la comunidad Cuatricentenaria del Municipio Pedraza, estado Barinas, Periodo escolar 2015-2016. Se inscribe en el enfoque cualitativo, bajo la modalidad de investigación acción participante sustentado en un diseño de campo. Para la recolección de la información se aplicó la técnica de la entrevista semiestructurada, Se contó con tres (03) informantes claves: Un (1) docente de aula, un (1) docente supervisor un (1) estudiante perteneciente al 4to año sección $\mathrm{E}$, para efectos de la comprobación, validación y fiabilidad requirió juicio de un (1) experto en planificación y evaluación, sobre el uso de estrategias transdisciplinaria. Para la presentación y análisis de resultados se categorizó, trianguló y teorizó la información obtenida. Se realizó un diagnostico detallado, del cual se diseñó la propuesta denominada "Estrategias para la ecología profunda y transdisciplinaria para la preservación de los ambientes educativos". Posteriormente se ejecutó la propuesta, en donde las actividades fueron catalogadas como importantes para brindar ambientes escolares agradables a la comunidad educativa. Concluyó que la Ecología Profunda debe ser Transdisciplinaria, una conexión entre todas las asignaturas, programas y haceres dentro y fuera de la Institución para lograr el objetivo que consiste en la transformación individual frente a los problemas ambientales.

Palabras clave: ecología profunda; transdisciplinaria; ambiente; conservación. 


\title{
Deep and Transdisciplinary Ecology in the Conservation of Educational Environments
}

\begin{abstract}
The present research work had as general purpose to develop actions of deep ecology and Transdisciplinary in the preservation of educational environments of the Bolivarian National Lyceum Dr. Carlos María González Bona, located in the community Cuatricentenaria of the Municipality Pedraza, Barinas state, School period 2015-2016. It is part of the qualitative approach, under the research modality participant action sustained in a field design. In order to collect the information, the technique of the semi-structured interview was applied. There were three (03) key informants: One (1) classroom teacher, one (1) teacher supervisor one (1), For purposes of verification, validation and reliability required judgment of one (1) planning and evaluation expert on the use of transdisciplinary strategies. For the presentation and analysis of results, the information obtained was categorized, triangulated and theorized. A detailed diagnosis was made, from which the proposal was named "Strategies for deep and transdisciplinary ecology for the preservation of educational environments". Subsequently, the proposal was implemented, where activities were classified as important to provide school environments that are pleasant to the educational community. He concluded that Deep Ecology should be Transdisciplinary, a connection between all subjects, programs and doings inside and outside the Institution to achieve the goal of individual transformation in the face of environmental problems.
\end{abstract}

Keywords: deep ecology; transdisciplinary; environment; conservation. 


\section{A Modo De Introducción}

La historia de la humanidad se ha transmitido de una generación a otra a través de la comunicación oral, sin embargo, es la escritura la que ha contribuido a la documentación de los grandes hechos que han cambiado al mundo. De allí surge la necesidad de crear mecanismos para la enseñanza de la historia, que a su vez es la cultura de cada pueblo. La educación surge como la alternativa masificar el acervo cultural de una nación, con el paso del tiempo este proceso se formalizó, con el objetivo de desarrollar políticas orientadas a los fines propuestos de los gobiernos. Las concepciones de la educación giran en torna al proceso de crear conocimientos, sobre la base dialógica de la enseña - aprendizaje.

Así mismo la evolución del conocimiento ha hecho de la educación un proceso mucho más dinámico, los métodos Han evolucionado pero el objetivo primordial es el mismo desde el inicio de los tiempos, el cual es el formar ciudadanos cónsonos con las exigencias de la sociedad donde se desenvuelve, en este sentido la educación ambiental es un proceso fundamental en la educación de las personas, para López, Morales y Potes (2015) la educación ambiental se define como "formación, a cualquier edad, de conceptos, valores y conductas, que ayudan al ciudadano a comprender el mundo que le rodea y del que depende, que tienden a prevenir los daños al ambiente (pág. 15). En este orden de ideas, el importante destacar que la educación ambiental es fundamental para formación integral de las personas.

En concordancia con lo expuesto con anterioridad, la educación ambiental ha sido abordada desde diversas perspectivas, en este estudio se hace referencia a la ecología profunda, enfoque que establece la necesidad de que el hombre se relacione de manera armónica con el ambiente, que se sienta parte de él y se responsabilice por sus acciones, según Aranda (2015);

Concibe y percibe al mundo no como una colección de objetos separados entre sí, sino como una compleja red de procesos 
interconectados e interdependientes que configuran una trama, de la cual los seres vi vos son una fibra (pág. 2).

De acuerdo con lo expresado por el autor antes citado es importante aclarar los términos relacionados con la ecología, porque se pretende ahondar, en principios y valores ecológicos fundamentales para la formación de una cultura ecológica, entendiéndola como un proceso educativo de carácter y no formal, continuo y necesario para el desarrollo de la vida.

Es por ello que se hace necesaria la inserción del pensamiento filosófico y educativo de ecología profunda en los estudiantes, como estrategia para la aceptación de cambio hacia los espacios donde principalmente radica su entorno educativo, es decir implicando la valoración de las condiciones ambientales como elemento que influye notoriamente en el aprendizaje del estudiante. Al respecto Speranza (2006), refiere que la ecología profunda y autorrealización:

Se destaca la importancia del contacto habitual con la naturaleza en el proceso formativo para fomentar el respeto por el medio ambiente. También se subraya la necesidad de transmitir que el cuidado de la naturaleza repercute en una mejora de la calidad de vida del hombre, ya que éste forma parte del entorno natural (pág. 6).

Lo antes expresado por Speranza, refleja la necesidad de transformación en la conducta del ser humano en formar su personalidad, sus actitudes para aumentar las fortalezas y disminuir las debilidades ante la desvalorización del ambiente que nos rodea en el campo educativo. Actualmente, en la educación media y diversificada, y en el Liceo Nacional Bolivariano Dr. Carlos María González Bona, municipio Pedraza, estado Barinas, se ha venido observando la ausencia de valoración hacia el entorno educativo por parte de la comunidad estudiantil, aunado a esto, de estrategias en las planificaciones que también subraye la necesidad de transmitir el 
cuidado de la naturaleza y del entorno donde vive el hombre, que repercuta en la mejora de la calidad educativa, ya que éste forma parte del hábitat cultural y ambiental.

Los educadores deben informar sobre las acciones concretas que se pueden realizar para mejorar el medio ambiente, fomentando el interés por el cambio continuo, sin limitaciones, la cual reforzará en el estudiante interés en la realización de actividades ecológicas, que conduzcan al éxito de la calidad de vida del ser humano al momento del cumplimiento de las actividades académicas, producto de la internalización con el ambiente que les rodea.

La poca interacción del estudiante hacia los docentes, al momento de realizar actividades que mejoren el ambiente y espacios educativos, también demuestra la ausencia de conciencia ecológica que obstaculiza el nuevo modelo de pensamiento en el ser humano. La cultura forjada desde los núcleos familiares (comunidad padres y representantes) hacia la preservación y conservación del entorno educativo, es carente, motivado al conocimiento vacío sobre la importancia de interrelación naturaleza-hombre, lo que afecta los espacios educativos.

Dicha realidad ya descrita, es lo que hace necesario desarrollar actividades que enfaticen la educación ambiental en el pensamiento estudiantil como método para el aprendizaje significativo, que garantice la preservación de los espacios institucionales, que puedan contribuir a la conservación de una naturaleza plena y holística al servicio de la comunidad. Que hagan prevalecer a favor del ecosistema y en los niveles de educación, afianzando compromisos que son adquiridos cognitivamente, a través de la interdisciplinariedad en la cual el docente debe proponer estrategias creativas que despierten interés, disfrute de actividades ecológicas perdurables en el futuro, dentro y fuera de la comunidad estudiantil. 


\subsection{Propósito de la investigación.}

Desarrollar acciones de Ecología Profunda y Transdisciplinaria en la Conservación de Ambientes Educativos, el Liceo Nacional Bolivariano Dr. Carlos María González Bona, ubicado en la comunidad Cuatricentenaria, del Municipio Pedraza, Estado Barinas, durante el periodo escolar 2015-2016.

\subsection{Antecedentes.}

Dentro los estudios considerados como antecedentes se encuentran el de Trejo y Marcano (2013), quienes realizaron un trabajo investigativo para la Universidad Pedagógica Experimental Libertador, Caracas. Titulado Propuesta de innovación con el uso de las TIC, para la promoción de Valores Ambientales en la Educación Primaria venezolana. Este estudio plantea que las Tic es una herramienta que representa el enriquecimiento que favorece el conocimiento dentro de las bases Transdisciplinarias, puesto que conduce a una estrategia innovadora y que en la actualidad es necesario para lograr el interés en la enseñanza de la Ecología Profunda y recuperación de los espacios educativos. La enseñanza de lo Transdisciplinario debe ser a la par de los cambios en la tecnología ya que los estudiantes se interesan por lo dinámico y no solamente en praxis que evidencian solo teorías o modos de actuar y de pensar.

Otro de los antecedentes lo constituye en trabajo de investigación realizado por Pérez (2013), denominado "Importancia de la acción individual en el fortalecimiento de la Educación Ambiental y la participación Ciudadana". Estudio realizado para la Universidad José Antonio Páez. Manifiesta la necesidad de involucrar al cambio y la transformación de los Espacios educativos no solamente a los estudiantes y docentes, dado que promover una transformación de valores es promovido por los Padres representantes que desde su hogar. Por otra parte, la comunidad en general debe promover la cooperación para conservar en el tiempo las instituciones que son la base de 
la plataforma cognoscitiva y axiológica de los individuos que reciben la enseñanza. De esta forma se logrará la transdisciplinariedad, debido a que el conocimiento y la praxis hacia la conservación trascienden hacia afuera, es decir involucra a la colectividad.

\section{Teoría y Conceptos.}

La presente investigación está orientada en el marco de la, teoría de la ecología profunda. La cual es definida por Hieronimi y Ortiz (2008) como "un enfoque holístico hacia el mundo, que une pensamiento sentimiento, espiritualidad y acción - Trata sobre como trascender el individualismo de la cultura occidental para vernos a nosotros mismos como parte de la tierra" (pág. 2). Es decir, que el comportamiento del individuo frente a los problemas ambientales será evaluado según su auto conciencia y participación ante la naturaleza, sin embargo, para lograr esto, se hace necesario motivar y transformar la conducta natural, que promuevan que la mente del hombre mejore su personalidad, partiendo desde los aprendizajes significativos.

\subsection{La transdisciplinariedad}

La transdisciplinariedad se presenta como una alternativa para optimizar la concepción de la Educación Media en nuestro contexto educativo, dentro de los escenarios de la complejidad. A la par de estas aseveraciones Morín (2002) señala: "La búsqueda de una Sociedad Mundo, formada por ciudadanos comprometidos en la construcción de una sociedad planetaria, en la cual impere el protagonismo, consciente y crítico, siendo el medio para alcanzarla, la educación." (pág. 34). Es así como esta investigación se convierte en la base del problema que dará una respuesta a la promoción de aprendizajes significativos y el éxito que juegan las estrategias para alcanzar dicho proceso transdisciplinario. 


\subsection{Beneficios de la transdisciplinariedad Ecologista.}

El desarrollo de la transdisciplinariedad ecologista en la Educación, ha favorecido la creación de grandes bases que permiten, simultáneamente, orientarse a una cultura ambientalista y de transformación. De allí que, en la dinámica estos elementos deben verse también de manera relacionada con la praxis en la transformación de espacios educativos. Promover una enseñanza transdisciplinaria en la preservación de los espacios educativos, fomenta prioritariamente las actividades de investigación, y concienciación con la ecología profunda en la percepción humanística del estudiante dentro y fuera del ámbito escolar. Al respecto Chaparro, (1998) comenta:

La tarea de la generación del conocimiento está asociada a la innovación, al compromiso que deben adquirir los estudiantes de aprender a lo largo de la vida y establecer redes que viabilicen el aprendizaje social. Esto lleva a una concepción dinámica de la relación entre el conocimiento, el sujeto que conoce y el entorno en el cual el mismo actúa (pág. 86)

De esta manera, el autor puede mencionar que las propuestas curriculares deben dar un rol privilegiado a la transdisciplinariedad, como acción necesaria del proceso de producción del conocimiento y como estrategia de enseñanza. Se requiere desarrollar proyectos de investigación que respondan a la dinámica que la ecología puede generar en los procesos de adquisición y difusión del conocimiento, consideración los procesos de gestión en las propias instituciones, a fin de sentar las bases para la gestión del conocimiento y favorecer el pensamiento conservacionista. Durante el proceso formativo en las instituciones educativas, se debe promover la conformación de grupos de trabajo e investigación cooperativos, integrados por diversidad de disciplinas, regiones geográficas e instituciones. 


\subsection{Teoría ecológica, fundamento de la educación.}

Con respecto a la relación entre la ecología y la educación, Según Barreno (2004) plantea que "El ambiente es, un aspecto muy importante para el desarrollo del ser humano, no se limita a entre un único entorno inmediato, sino que se extiende para incluir las interconexiones los entornos, y sus influencias externas." (pág. 22). Por este motivo, La Teoría Ecológica sostiene que el entorno natural al que pertenece el ser humano le ofrece una serie de posibilidades y oportunidades, pues, está inmersos en un ambiente natural que sufre explotación y abusos y que, de una u otra forma, aquello influye sobre el desarrollo del mismo.

Se debe entender que el ambiente natural referente al entorno escolar es un factor de desarrollo integral del ser humano y de la equidad social, pues éste es el que permite el desarrollo armónico de la sociedad en general, formando parte del desarrollo holístico inmerso en los aprendizajes. De igual manera, Barreno (ob.cit) sostiene que "La ecología del desarrollo humano comprende un continuo estudio de la Progresiva acomodación mutua entre el ser humano en desarrollo, y las propiedades cambiantes del entorno inmediato en donde vive cada persona" (pág. 123). Esto es debido a que no se considera a la persona en desarrollo como un individuo que repercute en el ambiente, sino como una entidad creciente, dinámica que va en desarrollo, Adentrándose progresivamente y reestructurando el medio en el que vive en un constante proceso de acomodación mutua, se considera que la interacción de la persona con el ambiente es bidireccional, es decir reciproco.

\subsection{Necesidad de la Educación Ambiental.}

En el Aspecto educativo, para encontrar solución a los problemas ambientales no es suficiente conformarnos con la teorización y los esquemas curriculares, sobre lo que acontece en el mundo en general y la problemática del medio ambiente en los niveles nacional y local. Adquirir nuevos modelos, 
actitudes y comportamientos humanos y adquirir nuevos conocimientos, y todo ello depende en gran medida de la educación.

Así mismo Barreno (2004), sostiene "La educación debe referirse a la toma de conciencia de los problemas ambientales y a que se los comprenda mejor, la educación debe ser permanente y abierta" (pág. 29). Es de esta manera como la autora enfatiza la necesidad acentuar progresivamente las acciones sobre las practicas ecológicas y la articulación de los contenidos basada en una conciencia ambiental y así lograr una compenetración entre la educación y la naturaleza, que no es más que los espacios educativos.

\section{Metodología.}

\subsection{Naturaleza del estudio, tipo y diseño.}

Este trabajo investigativo se enmarca bajo los parámetros de la investigación cualitativa la cual es definida por Sandín (2003) de la siguiente manera:

Investigación que produce resultados a los que no se ha llegado por procedimientos estadísticos $u$ otro tipo de cuantificación. Puede referirse a la investigación acerca de la vida de las personas, historias, comportamientos y también al funcionamiento organizativo...". (pág. 39).

Con respecto al diseño de investigación se adoptó la investigación acción participante, según Caicedo (2003) "es un estudio que surge a partir de un problema que se origina en la misma comunidad con el objeto de que en la búsqueda de la solución se mejore el nivel de vida de las personas involucradas" (pág. 40). Es por ello que la investigación acción es un método de estudio y acción de tipo cualitativo, que busca obtener resultados fiables y útiles para mejorar situaciones colectivas, que amerita una implicación y convivencia del personal técnico investigador en la comunidad a estudiar la 
acción para resolver el problema, su ejecución y evaluación de resultados, pasos que luego se repetían en forma reiterativa y cíclicas.

\subsection{Escenario e informantes Claves}

El escenario de la investigación estuvo constituido por el ámbito físico del Liceo nacional Bolivariano Dr. Carlos María González Bona en el Municipio Pedraza, Estado Barinas, con respecto a los informantes claves, para esta investigación se contó con tres (03) informantes claves. Un (1) docente de aula, un (1) docente supervisor un (1) estudiante perteneciente al 4to año sección "E". Los informantes seleccionados tienen la característica de ser las personas que viven la realidad de la institución, son los actores principales de este proceso, por lo cual ofrecen visiones diversas sobre el tema de la ecología profunda, lo cual es importante a la hora de contrastar las experiencias relatadas, los testimonios de los entrevistados son el sustento fundamental para la teorización del estudio.

\subsection{Técnicas e instrumentos de recolección de información.}

En este sentido, atendiendo a los objetivos del estudio, así como al contexto de investigación, las técnicas de recolección de información que se utilizaron fueron: la entrevista semiestructurada utilizada específicamente durante la realización del diagnóstico y la Observación Directa utilizada en todas las fases del estudio.

Esta técnica es una herramienta importante porque permite diferenciar la entrevista del discurso, del interrogatorio o de la conversación individual o grupal, ya que se trata de una forma estructurada de interacción que es artificial y planificada, dirigida a objetivos concretos, y que busca la objetividad y el control. En relación con la observación participante, siendo una técnica clásica para la adquisición de información, es de gran importancia durante el 
desarrollo de esta investigación porque permitió al investigador evidenciar lo más cerca posible las diversas realidades de los actores sociales.

En lo que respecta a esta investigación, se utilizó como instrumento una guía de entrevista semiestructurada contentiva de diez interrogantes, relacionadas la ecología profunda e interdisciplinaria, diseñada previamente por la investigadora, las cuales fueron formuladas posteriormente al entrevistado. La ventaja de este tipo de instrumento fue que permitió incorporar preguntas que inicialmente no estaban contempladas en el guión, facilitando el proceso de flexibilidad y confianza entre los agentes que interactúan directamente en el proceso.

\subsection{Presentación y análisis de la información}

La información suministrada será producto de aplicación de las diferentes técnicas de investigación, posteriormente se analizaron los resultados a través de la triangulación, Según Grijalva y Mancheno (2012):

Una de las técnicas de análisis de datos más características de la metodología cualitativa es la "triangulación". El principio básico consiste en recoger y analizar datos desde distintos ángulos para compararlos y contrastarlos entre sí. Denzin (1970: 291) define la triangulación como "la combinación de metodologías en el estudio de un mismo fenómeno". Para Kemmis (1983) consiste en un control cruzado entre diferentes fuentes de datos: personas, instrumentos, documentos o la combinación de todos ellos. (pág. 1)

En este orden de ideas, la información que se presenta, es el reflejo de la categorización, contrastación y teorización; constituyendo la elaboración de un diagnostico profundo y detallado de la situación en estudio, lo que le da a la investigación rigurosidad y consistencia. 


\subsection{Validez y Fiabilidad}

Martínez (2012) señala que "Una investigación con una buena confiabilidad es aquella que es estable, segura, congruente, igual a sí misma en diferentes tiempos y previsible para el futuro" (pág. 204). Para la confiabilidad se utilizarán los registros en cintas y filmaciones.

La validez interna, también es explicada por Martínez (2012) en otro de sus textos de estudio describiendo que "puede ser definida por el grado o nivel en que los resultados de la investigación reflejan una imagen clara y representativa de una realidad o situación dada”. (pág. 119). En este sentido, la fiabilidad está orientada hacia el nivel de concordancia interpretativa de los evaluadores de un mismo fenómeno, con el fin de sintetizar conclusiones y sugerencias; esto se logra adaptando los métodos con las técnicas a la naturaleza del estudio para evitar que se convierta en un hecho subjetivo sin un objetivo.

Tomando en cuenta a Ruiz Olabunaéga, J. (1996), para determinar los puntos coincidentes a partir de las diferentes fuentes informativas, La triangulación es un tipo de control de calidad que, en principio, es aplicado a este tipo de investigaciones cualitativas. Caracterizado Para efectos del presente trabajo la comprobación, validación y fiabilidad se basarán en el juicio de un (1) experto en planificación y evaluación, sobre el uso de estrategias trandisciplinarias apreciando al mismo tiempo el propósito y las circunstancias de investigación. En este sentido, los expertos consideraron las categorías predeterminadas (las cuales fueron mejoradas en el contexto abordado), junto a los aportes más resaltantes de los aspectos teóricos abordados.

En cuanto al procedimiento a seguir para la triangulación, también se requirió recabar toda la información posible de los tres (03) informantes seleccionados para luego ejecutar un cruce de datos que posibilite encontrar algunas similitudes y opiniones diferentes acerca del tema de la Ecología 
Profunda Transdisciplinaria en la preservación de los Ambientes Educativos. Caso Liceo Bolivariano Dr. Carlos María González Bona.

\section{Resultados.}

Los informantes manifestaron que en la educación Ambiental fomenta en el individuo sensibilización por el entorno natural desde las aulas de clase, enfrentando problemas de actitud pasiva por medio de los conocimientos y recobrando la sostenibilidad de los espacios educativos.

Asimismo, la investigación evidencio que la utilidad de la transdisciplinariedad en la formación eco ambiental comienza a partir de la Indagación de temáticas sobre problemas ambientales, promover planificadamente actividades para desarrollar nuevos patrones conservacionistas. Las personas entrevistadas opinaron que los modelos pedagógicos para lograr una educación Ambiental deben estar vinculados a estudiar temas de interés que satisfagan las necesidades sociales de alcance, utilizando estrategias novedosas como la tecnología y la dinámica.

Los informantes claves concuerdan que la evaluación Transdisciplinaria en la educación ambiental que debe estar integrada por la interacción social entre el docente y el estudiante para identificar potencialidades, intereses, visiones y recursos, llegando a apropiarse de la comprensión y de la significación como factores fundamentales de aprendizajes, reflexionando y valorando la conciencia individual y ambiental de los conocimientos teóricos prácticos de impacto.

El estudio concluyo que la evaluación Transdisciplinaria en la educación ambiental que debe estar integrada por la planificación del docente para lograr un aprendizaje en donde valore la importancia de la conservación de los ambientes articulando los contenidos y las experiencias. Los informantes opinaron que la ecología profunda corresponde a la conservación de los ambientes, aunque no corresponde con la teoría filosófica de Naess. 
Como hallazgo importante, los entrevistados estuvieron de acuerdo en que la ecología de los saberes consiste en el conocimiento amplio en diferentes temáticas, que se articulan entre sí, por medio de las distintas áreas del saber, que son necesarias en la solución de conflictos ambientales tomando en cuenta la experimentación y contacto con el entorno que nos rodea y la naturaleza. Los informantes denotan en sus aportes que la Ecología tiene inmerso un aprendizaje reflexivo que amplía su relación.

\section{Conclusiones}

La educación Ambiental fomenta en el individuo sensibilización por el entorno natural desde las aulas de clase, enfrentando problemas de actitud pasiva por medio de los conocimientos y recobrando la sostenibilidad de los espacios educativos.

La ecología Profunda y Transdisciplinaria, es una ciencia que promueve el aprendizaje en un proceso de adquisición para cambiar la percepción o la conducta hacia la conservación del ambiente como resultado de la experiencia, así como la interiorización en el que el estudiante concilia los nuevos conocimientos a sus estructuras cognitiva, a través del reconocimiento de todas las áreas del saber en el Liceo Nacional Bolivariano Dr. Carlos María González Bona. 2015- 2016.

La ecología Profunda y Transdisciplinaria para la conservación de los Ambientes educativos debe estar inmersa de estrategias comunitarias en el cual se preparen a los individuos para el dialogo de saberes y haceres en la formación y conservación del campo educativo en el Liceo Nacional Bolivariano Dr. Carlos María González Bona 2015-2016.

El uso de la tecnología TIC es una herramienta fundamental innovadora para promover la Conservación de los Espacios educativos a través de la Ecología Profunda Transdisciplinaria en el Liceo Nacional Bolivariano Dr. Carlos María González Bona 2015- 2016. 


\section{Referencias.}

Aranda, J. (2015). La alfabetización ecológica como nueva pedagogía para la comprensión de los seres vivientes. Colombia. Revista Luna Azul, Nro. 41. Recuperado de:

http://www.scielo.org.co/pdf/luaz/n41/n41a20.pdf

Barreno, L. (2004). Estrategias para promover la Educación Ambiental en niños y niñas de 4 a 5 años. Ecuador. Universidad tecnológica Equinoccial. Programa de Educación y Recreación.

Caicedo M. (2003). Compendio de Metodología Cualitativa en la Investigación Universidad Nacional Experimental de la Fuerza Armada. Caracas Venezuela.

Chaparro, P. (1998). Orientaciones para la transformación curricular Universitaria del siglo XXI. Coordinación general Universidad Carabobo, Venezuela.

Grijalva y Mancheno (2012). Investigación cualitativa. Recuperado de: http://www.monografias.com/trabajos95/investigacioncualitativa/investi gacioncualitativa.shtml

Hieronimi, H. y Ortiz, M. (2008). ¿Qué es ecología profunda?. Recuperado de: http://www.tierramor.org/EcologiaProfunda/EcoProfunda.html

Morín, E. (2003). Educar en Era planetaria. España Editorial Genisa, Sandín, M. (2003). "Investigación Cualitativa en Educación". Madrid, España.

Speranza, A. (2006). Ecología profunda y autorrealización, introducción a la ecología profunda de Arnés Naess. Buenos Aires. Editorial Biblios.

Trejo, C. y Marcano N. (2013). Propuesta de innovación Educativa mediante el uso de las TIC para la Promoción de valores ambientales en la Educación Primaria venezolana. Universidad Pedagógica Experimental Libertador, Caracas, Venezuela. 


\section{Janry Mercedes Nádales Pérez}

e-mail: janrynadales@gmail.com

Nacida en Ciudad Bolivia, Estado Barinas, Venezuela.

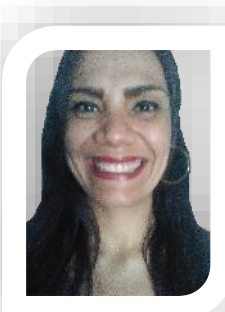

Cursa estudio de Maestría en Educación Ambiente y

Desarrollo en la Universidad Pedagógica Experimental

Libertador en Barinas Estado Barinas. Licenciada en

Educación Mención Historia. Universidad del Zulia.

Maracaibo Estado Zulia. Se desempeña como docente

de aula en el Liceo Nacional Bolivariano. Dr. Carlos María González Bona.

Ciudad Bolivia. Municipio Pedraza. Estado Barinas durante once años.

El contenido de este manuscrito se difunde bajo una Licencia de Creative Commons ReconocimientoNoComercial-Compartirlgual 4.0 Internacional 